\title{
Error estimates for parabolic optimal control problems with control constraints
}

\author{
Arnd Rösch
}

Preprint 2003/18

\section{Preprint-Reihe des Instituts für Mathematik Technische Universität Berlin}


An optimal control problem for the 1-d heat equation is investigated with pointwise control constraints. This paper is concerned with the discretization of the control by piecewise linear functions. The connection between the solutions of the discretized problems and the continuous one is investigated. Under an additional assumption on the adjoint state an approximation order $\sigma^{3 / 2}$ is proved for uniform discretizations. In the general case it is shown that a non-uniform control discretization ensure an approximation of order $\sigma^{3 / 2}$. Numerical tests confirm the theoretical part.

AMS subject classification: 49N10, 49M05, 65K10 


\title{
Error estimates for parabolic optimal control problems with control constraints
}

\author{
A. Rösch *
}

\begin{abstract}
An optimal control problem for the 1-d heat equation is investigated with pointwise control constraints. This paper is concerned with the discretization of the control by piecewise linear functions. The connection between the solutions of the discretized problems and the continuous one is investigated. Under an additional assumption on the adjoint state an approximation order $\sigma^{3 / 2}$ is proved for uniform discretizations. In the general case it is shown that a non-uniform control discretization ensure an approximation of order $\sigma^{3 / 2}$. Numerical tests confirm the theoretical part.
\end{abstract}

Keywords: Linear-quadratic optimal control problems, error estimates, heat equation, non-uniform grids, numerical approximation, control constraints.

AMS subject classification: 49N10, 49M05, 65K10

\section{Introduction}

The paper is concerned with the discretization of parabolic optimal control problems. We discuss here the case of a boundary control of the 1-d heat equation

$$
\begin{aligned}
y_{t}(t, x) & =y_{x x}(t, x) & & \text { in }(0, T) \times(0,1) \\
y(0, x) & =y^{o}(x) & & \text { in }(0,1) \\
y_{x}(t, 0) & =0 & & \text { in }(0, T) \\
y_{x}(t, 1) & =u(t) & & \text { in }(0, T)
\end{aligned}
$$

*Technische Universität Berlin, Fakultät II Mathematik und Naturwissenschaften, Straße des 17. Juni 136, D-10623 Berlin, Germany 


$$
(P)\left\{\begin{array}{l}
\min \quad J(u)=\frac{1}{2}\left\|y(T, .)-y_{d}\right\|_{Y}^{2}+\frac{\nu}{2}\|u\|_{U}^{2} \\
\text { subject to }(1.1) \\
\text { and } u \in C=\{u \in U \mid a \leq u \leq b \quad \text { a.e. on }[0, T]\}
\end{array}\right.
$$

where $U=L^{2}(0, T), Y=L^{2}(0,1)$, and $y^{o}, y_{d} \in Y$. Moreover, $\nu>0, a, b$ are real numbers with $a>b$. We only discuss the effect of the control discretization here. Therefore, we introduce the control discretized counterpart of $(P)$

$$
\left(P_{\sigma}\right)\left\{\begin{array}{l}
\min \quad J(u)=\frac{1}{2}\left\|y(T, .)-y_{d}\right\|_{Y}^{2}+\frac{\nu}{2}\|u\|_{U}^{2} \\
\text { subject to }(1.1) \\
\text { and } u \in C_{\sigma}=\left\{u \in U_{\sigma} \mid a \leq u \leq b \quad \text { a.e. on }[0, T]\right\} .
\end{array}\right.
$$

Here, the state space $Y$ is not discretized.

In this paper, we discuss a space $U_{\sigma}$ of piecewise linear functions on uniform and non-uniform grids.

Elliptic optimal control problems discretized by piecewise constant functions are well investigated, we refer to Falk [3], Geveci [4], and Arada/Casas/Tröltzsch [1]. The authors show for different examples the convergence order $\sigma$. The parabolic case with controls piecewise constant in time is discussed in Malanowski [12]. In that paper a convergence rate $\sigma^{1 / 2}$ is proved for parabolic optimal control problems with boundary control.

Two difficulties occur in our simple problem $(P)$ which are typical for parabolic optimal control problems. First, the optimal control does not belong to the space $H^{1}(0, T)$ in general. Therefore it is not possible to apply the results of Casas/Tröltzsch [2] or Rösch [13] directly. Hence, we cannot expect approximation order $\sigma$ or higher.

In [13] it is shown that in sufficiently regular cases the convergence rate $\sigma^{3 / 2}$ is obtained. We will describe situations where this approximation order for uniform grids holds in this paper. Moreover, the convergence rate $\sigma^{3 / 2}$ is proved for suitable non-uniform grids in a general case.

A completely different way is gone by Hinze [7]. In that approach only the state space is discretized. The optimal control can be obtained by projection of the adjoint state to the set of admissible controls. Therefore, the obtained optimal controls do not belong to finite dimensional subspaces of $U$.

Linear-quadratic optimal control problems are subproblems of SQP-algorithms and other higher order methods solving nonlinear optimal control problems, see for instance Heinkenschloss/Tröltzsch [6], Kelley/Sachs [8], Kunisch/Sachs [10], Tröltzsch [14] and the references therein.

The linear-quadratic optimal control problems can be attacked by a primal-dual active set strategy, see Hager [5] or Kunisch/Rösch [9]. The undiscretized optimal control problem can be solved theoretically using this strategy with an arbitrary 
high accuracy. Unfortunately, we are not able to solve the appearing system of equations exactly. Thus, it is necessary to discretize control and state. Therefore the approximation error of the solution of the discretized problem with respect to the continuous one plays an important role.

We describe the discretized space $U_{h}$ in the usual form: For a given grid $t_{i} \in[0, T]$ $(i=0 . . n)$ with $t_{0}=0$ and $t_{n}=T$ we define the functions $e_{i}$ by

$$
e_{i}= \begin{cases}\frac{x-t_{i-1}}{t_{i}-t_{i-1}} & \text { if } x \in\left[t_{i-1}, t_{i}\right) \\ \frac{t_{i+1}-x}{t_{i+1}-t_{i}} & \text { if } x \in\left[t_{i}, t_{i+1}\right] \\ 0 & \text { otherwise. }\end{cases}
$$

Now $u \in U_{\sigma}$ can be expressed as

$$
u=\sum_{i=0}^{n} u_{i} e_{i} .
$$

Moreover, $u \in C_{\sigma}$ means

$$
a \leq u_{i} \leq b \quad \forall i=0 . . n .
$$

Thus, $\left(P_{\sigma}\right)$ can be equivalently written as

$$
\left(P_{\sigma}^{\prime}\right)\left\{\begin{array}{l}
\min \quad J(u)=\frac{1}{2}\left\|y-y_{d}\right\|_{Y}^{2}+\frac{\nu}{2}\|u\|_{U}^{2} \\
\text { subject to } y=S u \\
u=\sum_{i=0}^{n} u_{i} e_{i} \\
\text { and } a \leq u_{i} \leq b \quad \forall i=0 . . n
\end{array}\right.
$$

in this case.

The paper is organized as follows: In section 2 we formulate the optimality conditions and state the main results. Section 3 contains several auxiliary results. The proof of the error estimates is presented in section 4 . The paper ends with numerical tests in section 5 .

\section{Optimality conditions}

First, we introduce the spaces $V=H^{1}(0,1)$ and $W(0, T)=\left\{v \in L^{2}(0, T ; V): v_{t} \in\right.$ $\left.L^{2}\left(0, T ; V^{*}\right)\right\}$. A weak solution $y \in W(0, T)$ is defined by the equations

$$
\begin{aligned}
\int_{0}^{1} y_{t} \cdot v d x+\int_{0}^{1} y_{x} \cdot v_{x} d x & =u \cdot v(1) \\
y(0) & =y^{o}
\end{aligned}
$$


for almost all $t$ and all $v \in V$. For the theory of weak solutions in $W(0, T)$, we refer to Lions/Magenes [11]

Lemma 1 For each $u \in L^{2}(0, T)$, equation (1.1) admits a unique solution $y \in$ $W(0, T)$. Hence, $y(T,$.$) belongs to Y$.

We introduce now the adjoint equation

$$
\begin{aligned}
-p_{t}(t, x) & =p_{x x}(t, x) & & \text { in }(0, T) \times(0,1) \\
p(T, x) & =y(T, x)-y_{d}(x) & & \text { in }(0,1) \\
p_{x}(t, 0) & =0 & & \text { in }(0, T) \\
p_{x}(t, 1) & =0 & & \text { in }(0, T) .
\end{aligned}
$$

Lemma 2 For each $y \in W(0, T)$, the adjoint equation (2.1) admits a unique solution $p \in W(0, T)$. Hence, $p(., 1)$ belongs to $U$.

Now we are able to formulate the necessary and sufficient optimality conditions for $(P)$ and $\left(P_{\sigma}\right)$.

Lemma 3 Let $\bar{u} \in C$ an admissible control for $(P)$ with associated state $\bar{y}$ and adjoint state $\bar{p}$ defined by (1.1) and (2.1). Then $\bar{u}$ is the optimal solution of $(P)$ if and only if

$$
(\bar{p}(., 1)+\nu \bar{u}, u-\bar{u})_{U} \geq 0
$$

holds for all $u \in C$. Analogue, let $u_{\sigma} \in C_{h}$ be an admissible control for $\left(P_{\sigma}\right)$ with associated state $\bar{y}$ and adjoint state $\bar{p}$ defined by (1.1) and (2.1). Then $u_{\sigma}$ is optimal for $\left(P_{\sigma}\right)$ if and only if

$$
\left(p_{\sigma}(., 1)+\nu u_{\sigma}, u-u_{\sigma}\right)_{U} \geq 0
$$

is fulfilled for all $u \in C_{\sigma}$.

The regularity of the adjoint state $\bar{p}$ plays an important role for error estimates. This regularity is influenced by the desired state $y_{d}$. Therefore, we require here an additional regularity assumption for $y_{d}$.

Assumption (A): The desired state $y_{d}$ belongs to $H^{2}(0,1)$.

Now we are able to state the main results of the paper:

Theorem 1 Suppose that

$$
-\frac{\bar{p}(T, 1)}{\nu} \notin[a, b]
$$


and assumption (A) holds. Moreover, $[0, T]$ is discretized uniformly $\left(t_{i}=i \cdot T / n\right)$. Then the estimate

$$
\left\|\bar{u}-u_{\sigma}\right\|_{U} \leq c \cdot \sigma^{3 / 2}
$$

is fulfilled for the optimal solutions $\bar{u}$ of $(P)$ and $u_{\sigma}$ of $\left(P_{\sigma}\right)$ with a positive constant $c>0$ and $\sigma=T / n$.

Theorem 2 Suppose that

$$
-\frac{\bar{p}(T, 1)}{\nu} \neq a, \quad-\frac{\bar{p}(T, 1)}{\nu} \neq b,
$$

and assumption $(A)$ holds. Moreover, $[0, T]$ is discretized in a suitable non-uniform way (for instance $t_{i}=T-T \cdot \frac{(n-i)^{4}}{n^{4}}(i=0 . . n)$ ). Then the estimate

$$
\left\|\bar{u}-u_{\sigma}\right\|_{U} \leq c \cdot \sigma^{3 / 2}
$$

is fulfilled for the optimal solutions $\bar{u}$ of $(P)$ and $u_{\sigma}$ of $\left(P_{\sigma}\right)$ with a positive constant $c>0$ and $\sigma=T / n$.

The proofs of Theorem 1 and Theorem 2 are contained in section 4 .

\section{Auxiliary results}

First, we introduce the Green's function

$$
G(t, x, \xi)=\sum_{n=0}^{\infty} v_{n}(x) v_{n}(\xi) e^{-n^{2} \pi^{2} t},
$$

where $v_{n}(x)=\sqrt{2} \cos n \pi x$ denote the normalized eigenfunctions of a Sturm-Liouville eigenvalue problem associated with the problem (1.1): $v_{x x}=\lambda v, v_{x}=0$ at $x=$ $0, x=1$.

For further investigations we need some estimates concerning the infinite sum in this function:

Lemma 4 For $0<t \leq T$ the estimates

$$
\begin{aligned}
\sum_{n=0}^{\infty} e^{-n^{2} \pi^{2} t} & \leq c t^{-1 / 2} \\
\sum_{n=0}^{\infty} n^{-2}\left(1-e^{-n^{2} \pi^{2} t}\right) & \leq c t^{1 / 2} \\
\sum_{n=0}^{\infty} n^{2} e^{-n^{2} \pi^{2} t} & \leq c t^{-3 / 2} \\
\sum_{n=0}^{\infty} n^{4} e^{-n^{2} \pi^{2} t} & \leq c t^{-5 / 2}
\end{aligned}
$$

hold true. 
Proof: We use a generic constant $c>0$

$$
\begin{aligned}
\sum_{n=0}^{\infty} e^{-n^{2} \pi^{2} t} & \leq 1+\sum_{n=1}^{\infty} e^{-n^{2} \pi^{2} t} \\
& \leq 1+\int_{0}^{\infty} e^{-x^{2} \pi^{2} t} d x \\
& \leq 1+c \cdot t^{-1 / 2} \\
& \leq c \cdot t^{-1 / 2}
\end{aligned}
$$

For the last inequality we used that $c \cdot t^{-1 / 2}>1$ on $(0, T]$ is fulfilled for a sufficiently large constant $c$. Therefore, estimate (3.2) holds true. Integrating this inequality, we obtain

$$
\int_{0}^{s} \sum_{n=0}^{\infty} e^{-n^{2} \pi^{2} t} d t \leq c \cdot s^{-1 / 2} .
$$

Changing integration and summation, we get

$$
\sum_{n=0}^{\infty}(\pi n)^{-2}\left(1-e^{-n^{2} \pi^{2} s}\right) \leq c s^{1 / 2} .
$$

The last two inequalities can be proved by similar arguments.

By the Fourier method, we get for the solution of (1.1)

$$
y(t, x)=\int_{0}^{t} G(t-s, x, 1) u(s) d s+\int_{0}^{1} G(t, x, \xi) y^{o}(\xi) d \xi .
$$

Using Green's function for the adjoint equation (2.1), we obtain the formula

$$
p(t, x)=\int_{0}^{1} G(T-t, x, \xi)\left(y(T, \xi)-y_{d}(\xi)\right) d \xi
$$

and in particular for the boundary values of the adjoint state yields

$$
p(t, 1)=\int_{0}^{1} G(T-t, 1, \xi)\left(y(T, \xi)-y_{d}(\xi)\right) d \xi .
$$

The boundary values of the optimal adjoint state $\bar{p}$ are directly coupled with the optimal control $\bar{u}$ via the formula

$$
\bar{u}=\Pi_{[a, b]}\left(-\frac{1}{\nu} \bar{p}(., 1)\right)
$$


with the projection operator

$$
\Pi_{[a, b]}(r)= \begin{cases}a & \text { for } r<a \\ r & \text { for } r \in[a, b] \\ b & \text { for } r>b\end{cases}
$$

This is a consequence of the optimality condition (2.2).

Next, we discuss the regularity of the optimal control and the optimal adjoint state.

Lemma 5 The optimal control $\bar{u}$ belongs to the space $C^{0,1 / 2}[0, T]$.

Proof: The proof is mainly standard. We sketch here only the main ideas. Inserting (3.6) in (3.7), we obtain

$$
\begin{aligned}
\bar{p}(t, 1)= & \int_{0}^{1} \int_{0}^{T} G(T-t, 1, \xi) G(T-s, \xi, 1) \bar{u}(s) d s d \xi \\
& +\int_{0}^{1} \int_{0}^{1} G(T-t, 1, \xi) G(T, \xi, \zeta) y^{o}(\zeta) d \zeta d \xi-\int_{0}^{1} G(T-t, 1, \xi) y_{d}(\xi) d \xi \\
= & \int_{0}^{T} \sum_{n=0}^{\infty} e^{-n^{2} \pi^{2}(2 T-t-s)} \bar{u}(s) d s+\int_{0}^{1} \sum_{n=0}^{\infty} e^{-n^{2} \pi^{2}(2 T-t)} v_{n}(\zeta) y^{o}(\zeta) d \zeta \\
& -\int_{0}^{1} \sum_{n=0}^{\infty} e^{-n^{2} \pi^{2}(T-t)} v_{n}(\xi) y_{d}(\xi) d \xi
\end{aligned}
$$

Hence, $\bar{p}(., 1)$ is analytic in every interval $[0, \alpha]$ with $\alpha<T$. For the regularity we investigate the crucial point $t=T$. We obtain for the difference $\bar{p}(T, 1)-\bar{p}(t, 1)$

$$
\begin{aligned}
\bar{p}(T, 1)-\bar{p}(t, 1)= & \int_{0}^{T} \sum_{n=0}^{\infty}\left(e^{-n^{2} \pi^{2}(T-s)}-e^{-n^{2} \pi^{2}(2 T-t-s)}\right) \bar{u}(s) d s \\
& +\int_{0}^{1} \sum_{n=0}^{\infty}\left(e^{-n^{2} \pi^{2} T}-e^{-n^{2} \pi^{2}(2 T-t)}\right) v_{n}(\zeta) y^{o}(\zeta) d \zeta \\
& -\int_{0}^{1} \sum_{n=0}^{\infty}\left(1-e^{-n^{2} \pi^{2}(T-t)}\right) v_{n}(\xi) y_{d}(\xi) d \xi
\end{aligned}
$$

The second term contains no singularity for $t \nearrow T$. Because of $y_{d} \in H^{2}(0,1)$ we have

$$
\left|\int_{0}^{1} v_{n}(\xi) y_{d}(\xi) d \xi\right| \leq \frac{c}{n^{2}} .
$$


From this estimate and (3.3), we get

$$
\left|\int_{0}^{1} \sum_{n=0}^{\infty}\left(1-e^{-n^{2} \pi^{2}(T-t)}\right) v_{n}(\xi) y_{d}(\xi) d \xi\right| \leq c \sqrt{T-t}
$$

It remains the first term. Using the positivity of each addend, we obtain

$$
\left|\int_{0}^{T} \sum_{n=0}^{\infty}\left(e^{-n^{2} \pi^{2}(T-s)}-e^{-n^{2} \pi^{2}(2 T-t-s)}\right) \bar{u}(s) d s\right| \leq c_{m} \int_{0}^{T} \sum_{n=0}^{\infty}\left(e^{-n^{2} \pi^{2}(T-s)}-e^{-n^{2} \pi^{2}(2 T-t-s)}\right) d s
$$

with $c_{m}=\max (|a|,|b|)$. We continue by

$$
\begin{aligned}
\left.\mid \int_{0}^{T} \sum_{n=0}^{\infty} e^{-n^{2} \pi^{2}(T-s)}-e^{-n^{2} \pi^{2}(2 T-t-s)}\right) \bar{u}(s) d s \mid \leq & c_{m} \int_{0}^{T} \sum_{n=0}^{\infty} e^{-n^{2} \pi^{2}(T-s)} d s \\
& -c_{m} \int_{t-T}^{t} \sum_{n=0}^{\infty} e^{-n^{2} \pi^{2}(T-s)} d s \\
& =-c_{m} \int_{t-T}^{0} \sum_{n=0}^{\infty} e^{-n^{2} \pi^{2}(T-s)} d s \\
& +c_{m} \int_{t}^{T} \sum_{n=0}^{\infty} e^{-n^{2} \pi^{2}(T-s)} d s \\
& \leq \quad+c_{m} \int_{t}^{T} \frac{c}{\sqrt{T-s}} d s \\
\leq & c \sqrt{T-t}
\end{aligned}
$$

using inequality (3.2) in the last step. Combining the results, we end up with

$$
|\bar{p}(T, 1)-\bar{p}(t, 1)| \leq c \sqrt{T-t}
$$

that implies

$$
|\bar{u}(T)-\bar{u}(t)| \leq c \sqrt{T-t}
$$

because of (3.8).

Remark 1 The result of Lemma 5 can also be proved for free or unilateral constrained parabolic optimal control problems. In this case, it has to be proved the boundedness of the optimal control in a first step.

Remark 2 Compatibility conditions have to be fulfilled for higher regularity of $\bar{p}(., 1)$, especially

$$
\left(\bar{y}(T, .)-y_{d}\right)_{x}(1)=0 .
$$

We can not expect this property in general. 
Remark 3 It is possible to prove that $\bar{p}(., 1)$ belongs to the Sobolev space $H^{1-\varepsilon}(0, T)$ for $\varepsilon>0$ using similar arguments like in the proof of Lemma 5. Again, the compatibility condition is needed for higher regularity.

The properties of $\bar{p}(., 1)$ are transferred to $\bar{u}$ by the projection formula (3.8). Clearly, $\bar{u}$ is smoother than $\bar{p}(., 1)$ if the projection cut the singular behaviour in a neighbourhood of $T$. This will be the key point in the proof of Theorem 1 in the next section. The situation is much more complicate if the projection is simply the identity. For this purpose, we formulate the next lemma.

Lemma 6 The function $\bar{p}(t, 1)$ is twice differentiable with respect to $t$ in $[0, T)$. Moreover, it holds

$$
\left|\bar{p}_{t t}(t, 1)\right| \leq c|T-t|^{-3 / 2}
$$

Proof: We start again with the formula

$$
\begin{aligned}
\bar{p}(t, 1)= & \int_{0}^{T} \sum_{n=0}^{\infty} e^{-n^{2} \pi^{2}(2 T-t-s)} \bar{u}(s) d s+\int_{0}^{1} \sum_{n=0}^{\infty} e^{-n^{2} \pi^{2}(2 T-t)} v_{n}(\zeta) y^{o}(\zeta) d \zeta \\
& -\int_{0}^{1} \sum_{n=0}^{\infty} e^{-n^{2} \pi^{2}(T-t)} v_{n}(\xi) y_{d}(\xi) d \xi
\end{aligned}
$$

Of course, $p(t, 1)$ is analytic in every interval $[0, \alpha]$ with $\alpha<T$. We differentiate twice $(t<T)$ and obtain

$$
\begin{aligned}
\bar{p}_{t t}(t, 1)= & \int_{0}^{T} \sum_{n=0}^{\infty} n^{4} \pi^{4} e^{-n^{2} \pi^{2}(2 T-t-s)} \bar{u}(s) d s+\int_{0}^{1} \sum_{n=0}^{\infty} n^{4} \pi^{4} e^{-n^{2} \pi^{2}(2 T-t)} v_{n}(\zeta) y^{o}(\zeta) d \zeta \\
& -\int_{0}^{1} \sum_{n=0}^{\infty} n^{4} \pi^{4} e^{-n^{2} \pi^{2}(T-t)} v_{n}(\xi) y_{d}(\xi) d \xi
\end{aligned}
$$

To get estimate (3.10), we have to estimate the absolute values of these three integrals. The first integral can be estimated using (3.5) since $u$ is pointwise bounded. The second integral has no singularity at $T$. It remains the last integral. Assumption (A) implies

$$
\left|\int_{0}^{1} v_{n}(\xi) y_{d}(\xi) d \xi\right| \leq c \cdot n^{2}
$$

Therefore we can use (3.4) to estimate the third integral. Consequently, we obtain

$$
\left|\bar{p}_{t t}(t, 1)\right| \leq c \cdot \int_{0}^{T}(2 T-t-s)^{-5 / 2} d t+c+c \cdot(T-t)^{-3 / 2} \leq c|T-t|^{-3 / 2}
$$


and the assertion is proved.

Clearly, estimate (3.10) is transferred to the optimal control $\bar{u}$ excepted the nondifferentiability points of the projection term. We are now able to prove a first approximation result.

Lemma 7 Let the discretization $t_{i}=T-T \cdot \frac{(n-i)^{4}}{n^{4}}(i=0 . . n)$ be given. Furthermore, let $v=\sum_{i=0}^{n} \bar{p}\left(t_{i}\right) e_{i}$ be the linear interpolate of $\bar{p}$. Then, the estimate

$$
\|v-\bar{p}(., 1)\|_{U} \leq \frac{c}{n^{2}}
$$

holds true.

Proof: Let $i<n$. Then, in every interval $\left[t_{i-1}, t_{i}\right]$ we have

$$
\bar{p}\left(t_{i-1}, 1\right)=v\left(t_{i-1}\right) \quad \text { and } \quad \bar{p}\left(t_{i}, 1\right)=v\left(t_{i}\right) .
$$

From this, we find easily

$$
|\bar{p}(t, 1)-v(t)| \leq c \max _{s \in\left[t_{i-1}, t_{i}\right]}\left|\bar{p}_{t t}(s, 1)\right|\left|t_{i}-t_{i-1}\right|^{2} \quad \forall t \in\left[t_{i-1}, t_{i}\right]
$$

Using (3.10), we obtain

$$
|\bar{p}(t, 1)-v(t)| \leq c\left(T-t_{i}\right)^{-3 / 2}\left|t_{i}-t_{i-1}\right|^{2} \quad \forall t \in\left[t_{i-1}, t_{i}\right] .
$$

Therefore, we can estimate the integral

$$
\int_{t_{i-1}}^{t_{i}}(\bar{p}(t, 1)-v(t))^{2} d t \leq c\left(T-t_{i}\right)^{-3}\left|t_{i}-t_{i-1}\right|^{4} d t \leq c\left(T-t_{i}\right)^{-3}\left|t_{i}-t_{i-1}\right|^{5} .
$$

We continue by

$$
\begin{aligned}
\|v-\bar{p}(., 1)\|_{U}^{2} & =\int_{0}^{T}(\bar{p}(t, 1)-v(t))^{2} d t \\
& \leq \sum_{i=1}^{n-1} \int_{t_{i-1}}^{t_{i}}(\bar{p}(t, 1)-v(t))^{2} d t+\int_{t_{n-1}}^{t_{n}}(\bar{p}(t, 1)-v(t))^{2} d t
\end{aligned}
$$

Because of the continuity of $\bar{p}$ we can estimate the second integral by

$$
\int_{t_{n-1}}^{t_{n}}(\bar{p}(t, 1)-v(t))^{2} d t \leq c\left(t_{n}-t_{n-1}\right)=\frac{c}{n^{4}} .
$$

It remains to discuss the sum in (3.12).

$$
\begin{aligned}
\sum_{i=1}^{n-1} \int_{t_{i-1}}^{t_{i}}(\bar{p}(t, 1)-v(t))^{2} d t & \leq c \cdot \sum_{i=1}^{n-1}\left(T-t_{i}\right)^{-3}\left|t_{i}-t_{i-1}\right|^{5} \\
& \leq c \cdot \sum_{i=1}^{n-1}\left(T\left(\frac{n-(i-1)}{n}\right)^{4}-T\left(\frac{n-i}{n}\right)^{4}\right)^{5}\left(T \cdot\left(\frac{n-i}{n}\right)^{4}\right)^{-3}
\end{aligned}
$$


Changing the summation order we obtain

$$
\begin{aligned}
\sum_{i=1}^{n-1} \int_{t_{i-1}}^{t_{i}}(\bar{p}(t, 1)-v(t))^{2} d t & \leq c \cdot \sum_{i=1}^{n-1}\left(T\left(\frac{i+1}{n}\right)^{4}-T\left(\frac{i}{n}\right)^{4}\right)^{5}\left(T \cdot\left(\frac{i}{n}\right)^{4}\right)^{-3} \\
& \leq c \cdot \sum_{i=1}^{n-1}\left(T \frac{4 i^{3}+6 i^{2}+4 i+1}{n^{4}}\right)^{5}\left(T \cdot\left(\frac{i}{n}\right)^{4}\right)^{-3} \\
& \leq c \cdot \sum_{i=1}^{n-1} \frac{1}{n^{8}} T^{5}(4+6+4+1)^{5} i^{15} \cdot i^{-12} T^{-3} \\
& \leq c \cdot \frac{1}{n^{4}}
\end{aligned}
$$

Inserting (3.14) and (3.13) in (3.12), we end up with

$$
\|v-\bar{p}(., 1)\|_{U}^{2} \leq c \cdot \frac{1}{n^{4}}
$$

that implies the assertion.

\section{Error estimates}

First, we recall a result of Casas/Tröltzsch [2]. For this purpose we define a function via

$$
v_{\sigma}^{i}= \begin{cases}a & \text { if } \min _{t \in\left[t_{i-1}, t_{i+1}\right]} \bar{u}(t)=a \\ b & \text { if } \max _{t \in\left[t_{i-1}, t_{i+1}\right]} \bar{u}(t)=b \\ \bar{u}\left(t_{i}\right) & \text { else }\end{cases}
$$

and

$$
v_{\sigma}=\sum_{i=0}^{n} v_{\sigma}^{i} e_{i} .
$$

If the mesh size $\sigma$ is sufficiently small, then $\bar{u}(t)=a$ and $\bar{u}\left(t^{\prime}\right)=b$ can not happen in the same interval $\left[t_{i-1}, t_{i+1}\right]$.

Lemma 8 (Casas/Tröltzsch [2]) The function $v_{\sigma}$ fulfil the condition

$$
\left(\bar{p}+\nu \bar{u}, v-v_{\sigma}\right)_{U} \geq 0
$$

for all $v \in C_{\sigma}$. Moreover, it holds

$$
\left\|\bar{u}-u_{\sigma}\right\|_{U} \leq c\left\|\bar{u}-v_{\sigma}\right\|_{U} .
$$


For the proof we refer to [2] Lemma 2.1 and the discussion afterwards.

Now, we are able to prove the main results presented in section 2.

Proof of Theorem 1: Here we have a uniform discretization and $\sigma=\frac{1}{n}$. We start with condition (2.4). Because of the continuity of $\bar{p}$, there exists an interval $\left[t^{\prime}, T\right]$

$$
-\frac{\bar{p}(t, 1)}{\nu} \notin[a, b] \quad \forall t \in\left[t^{\prime}, T\right]
$$

From (3.8), we know that this interval belongs to the set of active constraints and we have $\bar{u} \equiv a$ or $\bar{u} \equiv b$ on $\left[t^{\prime}, T\right]$. On the other interval $\left[0, t^{\prime}\right]$ the adjoint state $\bar{p}(., 1)$ is analytic.

Next, we estimate the term $\left\|\bar{u}-v_{\sigma}\right\|_{U}$, where $v_{\sigma}$ is the function defined above. For that purpose we subdivide the intervals $\left[t_{i-1}, t_{i}\right]$ in three disjoint classes

$$
\begin{aligned}
& I_{1}:=\left\{i \in\{1, . ., n\}: v_{\sigma}\left(t_{i-1}\right), v_{\sigma}\left(t_{i}\right) \in(a, b)\right\}, \\
& I_{2}:=\left\{i \in\{1, . ., n\}: \bar{u}(t) \equiv a \text { or } \bar{u}(t) \equiv b \text { on }\left[t_{i-1}, t_{i}\right]\right\}, \\
& I_{3}:=[0, T] \backslash\left(I_{1} \cup I_{2}\right) .
\end{aligned}
$$

We continue by

$$
\left\|\bar{u}-v_{\sigma}\right\|_{U}^{2}=\left\|\bar{u}-v_{\sigma}\right\|_{L^{2}\left(I_{1}\right)}^{2}+\left\|\bar{u}-v_{\sigma}\right\|_{L^{2}\left(I_{2}\right)}^{2}+\left\|\bar{u}-v_{\sigma}\right\|_{L^{2}\left(I_{3}\right)}^{2} .
$$

By definition we obtain

$$
\left\|\bar{u}-v_{\sigma}\right\|_{L^{2}\left(I_{2}\right)}^{2}=0 .
$$

On $I_{1}$, the function $v_{\sigma}$ is the linear interpolate of $\bar{u}$. Moreover, on this set $\bar{u}$ is analytic and belongs therefore especially to $C^{2}$. Hence we obtain

$$
\left\|\bar{u}-v_{\sigma}\right\|_{L^{2}\left(I_{1}\right)}^{2} \leq c \cdot \sigma^{4}
$$

It remains to estimate the last norm. The set $I_{3}$ can intersect the interval $\left[t^{\prime}, T\right]$. For sufficiently fine discretization, it is possible to find a $t^{\prime \prime}$ with $t^{\prime} \leq t^{\prime \prime}<T$ such that $I_{3} \cap\left[t^{\prime \prime}, T\right]=\emptyset$. Since $\bar{p}(., 1)$ is analytic on $\left[0, t^{\prime \prime}\right]$, there exist only finitely many points with $-\frac{1}{\nu} \bar{p}(t, 1)=a$ or $-\frac{1}{\nu} \bar{p}(t, 1)=b$ on $\left[0, t^{\prime}\right]$. Therefore, the number of intervals $\left[t_{i-1}, t_{i}\right]$ which are subsets of $I_{3}$ can be bounded by a finite number $K$ independent of the discretization. The optimal control $\bar{u}$ is lipschitz continuous on $I_{3}$ and we can estimate

$$
\left\|\bar{u}-v_{\sigma}\right\|_{L^{2}\left(I_{3}\right)}^{2}=\int_{I_{3}}\left(\bar{u}-v_{\sigma}\right)^{2} d t \leq c K L^{2} \cdot \sigma^{3} .
$$

From this, we get

$$
\left\|\bar{u}-v_{\sigma}\right\|_{U} \leq c \cdot \sigma^{3 / 2}
$$

and formula (4.2) implies the assertion. 
In this proof, we benefit from the active control part at the end of the interval $[0, T]$. If inequality (2.4) is not valid, then the projection formula (3.8) does not cut the problematic part at the end of the time interval. It may happen that the set $I_{1}$ contains an interval $\left[t^{*}, T\right]$. From section 3 , we know that $\bar{u}$ is in general only $C^{1 / 2}[0, T]$ (Lemma 5) or $H^{1-\varepsilon}(0, T)$. Applying the same technique as in the proof before, we obtain:

Remark 4 Let assumption (A) be fulfilled and let inequality (2.4) not be valid. Then an error estimate

$$
\left\|\bar{u}-u_{\sigma}\right\|_{U} \leq c \cdot \sigma^{1-\varepsilon}
$$

is obtained with $\varepsilon>0$ for uniform grids.

In this case a non-uniform grid can improve the convergence rate. In the proof of Theorem 2 we combine the technique of the last proof with the result of Lemma 7.

Proof of Theorem 2: First, we find for the non-uniform grid $t_{i}=T-T \cdot \frac{(n-i)^{4}}{n^{4}}(i=$ $0 . . n)$ :

$$
\sigma=\max _{i=1 . . n}\left|t_{i}-t_{i-1}\right|=t_{1}-t_{0} \leq \frac{4}{n}
$$

It is easy to see that $\sigma \geq \frac{3}{n}$ for sufficiently large $n$. We have to discuss two cases. In the first case, inequality (2.4) is fulfilled. Here, we can directly apply the arguments of the proof of Theorem 1 to obtain the assertion. In the second case, we have

$$
-\frac{1}{\nu} \bar{p}(T, 1) \in(a, b)
$$

Consequently, there exists an interval $\left[t^{*}, T\right]$ with

$$
-\frac{1}{\nu} \bar{p}(t, 1) \in(a, b) \quad \text { for all } t \in\left[t^{*}, T\right] .
$$

Without lost of generality, let $t^{*}$ be a grid point. Next, we change the definition of the sets $I_{1}, I_{2}, I_{3}$ a little bit. We use the same definition but replace $T$ by $t^{*}$. On this interval we can apply the argumentation of the proof of Theorem 1 and obtain

$$
\left\|\bar{u}-v_{\sigma}\right\|_{L^{2}\left(0, t^{*}\right)}^{2} \leq c \cdot \sigma^{3} .
$$

For the interval $\left[t^{*}, T\right]$ we find

$$
\left\|\bar{u}-v_{\sigma}\right\|_{L^{2}\left(t^{*}, T\right)}^{2}=\left\|-\frac{1}{\nu} \bar{p}(., 1)+\frac{1}{\nu} v\right\|_{L^{2}\left(t^{*}, T\right)}^{2},
$$

where $v$ is the linear interpolate of $\bar{p}(., 1)$ defined in Lemma 7 . Now, Lemma 7 implies

$$
\left\|\bar{u}-v_{\sigma}\right\|_{L^{2}\left(t^{*}, T\right)}^{2} \leq \frac{1}{\nu^{2}}\|\bar{p}(., 1)-v\|_{U}^{2} \leq \frac{c}{n^{4}} \leq c \cdot \sigma^{4} .
$$

Therefore, we have

$$
\left\|\bar{u}-v_{\sigma}\right\|_{U} \leq c \cdot \sigma^{3 / 2} .
$$

and again the assertion follows from inequality (4.2). 


\section{$5 \quad$ Numerical Tests}

In this section we present some illustrating numerical tests confirming the theoretical results. The linear-quadratic optimal control problems were solved by a primal-dual active set strategy, see Hager [5] or Kunisch/Rösch [9]. The parabolic equations are discretized by a Crank-Nicolson scheme. The space discretization in all numerical examples is $n_{x}=250$.

Example 1: This example is constructed in such a way that no constraint is active.

$$
T=1.58, \quad \nu=0.01, \quad y_{d}=\frac{1}{2}\left(1-x^{2}\right), \quad a=-10, \quad b=10
$$

First, we present the uniform discretization. We compare all solutions with $\hat{u}=$ $u_{1024}$. The last column contains the value

$$
\text { rate }:=\frac{\ln \left\|u_{n}-\hat{u}\right\|-\ln \left\|u_{512}-\hat{u}\right\|}{\ln 512-\ln n}
$$

which is an approximation of the convergence rate.

\begin{tabular}{|r|c|c|c|c|}
\hline$n$ & $\left\|u_{n}-\hat{u}\right\|_{U}$ & $n \cdot\left\|u_{n}-\hat{u}\right\|_{U}$ & $n^{2} \cdot\left\|u_{n}-\hat{u}\right\|_{U}$ & rate \\
\hline 8 & 0.0687 & 0.5496 & 4.3966 & 1.01482 \\
16 & 0.0670 & 1.0727 & 17.1632 & 1.21075 \\
32 & 0.0346 & 1.1067 & 35.4138 & 1.27469 \\
64 & 0.0115 & 0.7359 & 47.0956 & 1.17001 \\
128 & 0.0026 & 0.3270 & 95.3775 & 0.66998 \\
256 & 0.0015 & 0.3726 & 300.2653 & 0.52810 \\
512 & 0.0010 & 0.5167 & 264.5663 & - \\
\hline
\end{tabular}

The convergence is at most linear. The next table shows the numerical result for the non-uniform grid.

\begin{tabular}{|r|c|c|c|c|}
\hline$n$ & $\left\|u_{n}-\hat{u}\right\|_{U}$ & $n \cdot\left\|u_{n}-\hat{u}\right\|_{U}$ & $n^{2} \cdot\left\|u_{n}-\hat{u}\right\|_{U}$ & rate \\
\hline 8 & 0.052232 & 0.4179 & 3.3429 & 2.06228 \\
16 & 0.013005 & 0.2081 & 3.3292 & 2.07355 \\
32 & 0.003242 & 0.1037 & 3.3199 & 2.09093 \\
64 & 0.000808 & 0.0517 & 3.3094 & 2.11972 \\
128 & 0.000200 & 0.0256 & 3.2743 & 2.17188 \\
256 & 0.000048 & 0.0122 & 3.1356 & 2.28133 \\
512 & 0.000010 & 0.0050 & 2.5801 & - \\
\hline
\end{tabular}


The convergence rate is here quadratic. This numerical result confirms the theoretical results due to the fact $I_{3}=\emptyset$. The numerical error for the non-uniform grid for 64 intervals is smaller than for the uniform grid with 512 intervals.

The solutions for $n=16$ and $n=32$ are plotted in figure 1 . These pictures show the problems of the uniform grids to fit the solution part at the end of the time interval.
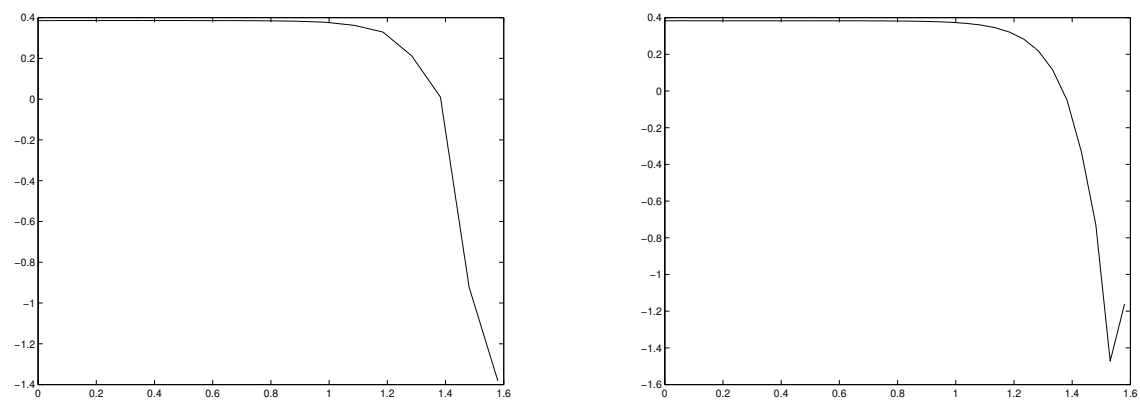

Figure 1: Uniform grids $n=16$ and $n=32$

Figure 2 illustrate the adapted behaviour of the non-uniform mesh. The grid $n=16$ (left picture) describes the solution part at the end of the time interval in a sufficient manner. The right picture shows the solution for $n=1024$.
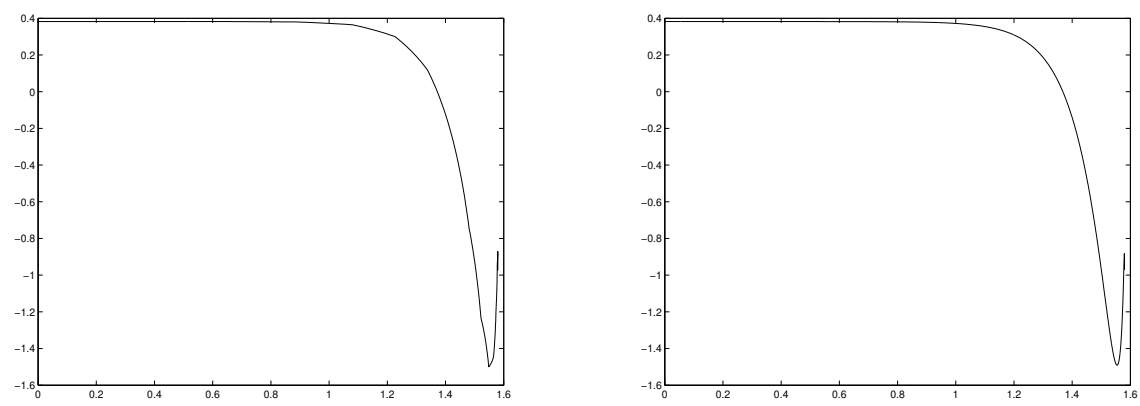

Figure 2: Non-uniform grid $n=16$ and $n=1024$

Example 2: This example is a real challenge for the non-uniform grid. Again, the solution oszillates heavily at the end of the time interval. The data are chosen in such a way that no constraint is active.

$$
T=1.58, \quad \nu=0.0001, \quad y_{d}=\frac{1}{2}(1-x), \quad a=-10, \quad b=10
$$

The next table show the quadratic convergence rate for the non-uniform grid. 


\begin{tabular}{|r|c|c|c|c|}
\hline$n$ & $\left\|u_{n}-\hat{u}\right\|_{U}$ & $n \cdot\left\|u_{n}-\hat{u}\right\|_{U}$ & $n^{2} \cdot\left\|u_{n}-\hat{u}\right\|_{U}$ & rate \\
\hline 8 & 0.248817 & 1.9905 & 15.9243 & 1.91795 \\
16 & 0.121227 & 1.9396 & 31.0341 & 2.09406 \\
32 & 0.027076 & 0.8664 & 27.7261 & 2.07692 \\
64 & 0.006543 & 0.4187 & 26.7984 & 2.08620 \\
128 & 0.001611 & 0.2062 & 26.3908 & 2.11824 \\
256 & 0.000390 & 0.0999 & 25.5747 & 2.19116 \\
512 & 0.000086 & 0.0438 & 22.4008 & - \\
\hline
\end{tabular}

The optimal solution for $n=16$ and $n=1024$ is plotted in Figure 3 .
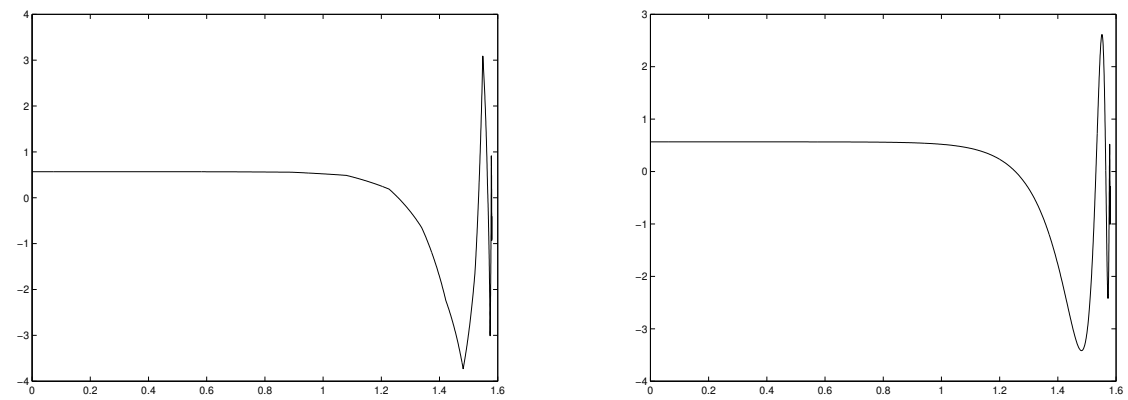

Figure 3: Non-uniform grid $n=16$ and $n=1024$

Example 3: This example illustrates the fact that active control constraints improve the convergence rate for uniform grids. The difference between the Examples 1 and 3 is the choice of $a$.

$$
T=1.58, \quad \nu=0.01, \quad y_{d}=\frac{1}{2}\left(1-x^{2}\right), \quad a=0, \quad b=10
$$

In the next table we slightly change the presentation of the numerical results.

\begin{tabular}{|r|c|c|c|c|}
\hline$n$ & $\left\|u_{n}-\hat{u}\right\|_{U}$ & $n \cdot\left\|u_{n}-\hat{u}\right\|_{U}$ & $n^{3 / 2} \cdot\left\|u_{n}-\hat{u}\right\|_{U}$ & $n^{2} \cdot\left\|u_{n}-\hat{u}\right\|_{U}$ \\
\hline 8 & 0.0083496 & 0.0668 & 0.1889 & 0.5344 \\
16 & 0.0068280 & 0.1092 & 0.4370 & 1.7480 \\
32 & 0.0040008 & 0.1280 & 0.7442 & 4.0968 \\
64 & 0.0005978 & 0.0383 & 0.3060 & 2.4485 \\
128 & 0.0003959 & 0.0507 & 0.5733 & 6.4859 \\
256 & 0.0001814 & 0.0464 & 0.7431 & 11.8889 \\
512 & 0.0000064 & 0.0033 & 0.0745 & 1.6845 \\
\hline
\end{tabular}

The table shows clearly the non-uniform convergence behaviour. This effect can be easily declared. In the proof of Theorem 1, we have a quadratic convergence order on the sets $I_{1}$ and $I_{2}$. In contrast to this, the convergence order on the set $I_{3}$ is only 
linear. Therefore, the $I_{3}$-part influences the convergence heavily. If we refine the grid we have two cases. In the first case, the smallest distance between the corner of the optimal control and a grid point does not change or change insignificant. In the second case, a new grid point fits the corner much better than all points of the coarser grid. These two cases lead to faster and slower phases in the convergence process. In the first two examples, we calculated convergence rates. Here, we abstain from a presentation of such rates, because a change from 512 to 256 in formula (4.1) causes to large differences in these rates.

The next figure shows the optimal solution for $n=16$ and $n=1024$.
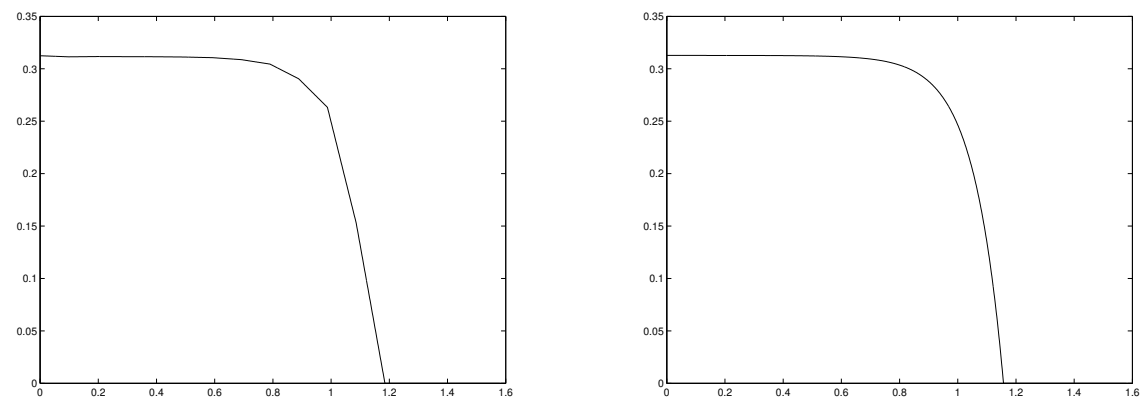

Figure 4: Non-uniform grid $n=16$ and $n=1024$

The next table shows the results for the non-uniform grid. The refinement at the end of the interval is in this case useless.

\begin{tabular}{|r|c|c|c|c|}
\hline$n$ & $\left\|u_{n}-\hat{u}\right\|_{U}$ & $n \cdot\left\|u_{n}-\hat{u}\right\|_{U}$ & $n^{3 / 2} \cdot\left\|u_{n}-\hat{u}\right\|_{U}$ & $n^{2} \cdot\left\|u_{n}-\hat{u}\right\|_{U}$ \\
\hline 8 & 0.0482019 & 0.3856 & 1.0907 & 3.0849 \\
16 & 0.0181645 & 0.2906 & 1.1625 & 4.6501 \\
32 & 0.0017108 & 0.0547 & 0.3097 & 1.7519 \\
64 & 0.0004213 & 0.0270 & 0.2157 & 1.7257 \\
128 & 0.0002350 & 0.0301 & 0.3403 & 3.8504 \\
256 & 0.0001613 & 0.0413 & 0.6608 & 10.5720 \\
512 & 0.0000954 & 0.0488 & 1.1048 & 24.9976 \\
\hline
\end{tabular}

The numerical solutions for $n=16$ and $n=32$ are plotted in the next figure. Here, the convergence rate of the non-uniform grid and the uniform grid is the same. Nevertheless, the uniform grid produces a slightly better solution for the same discretization in this case. 

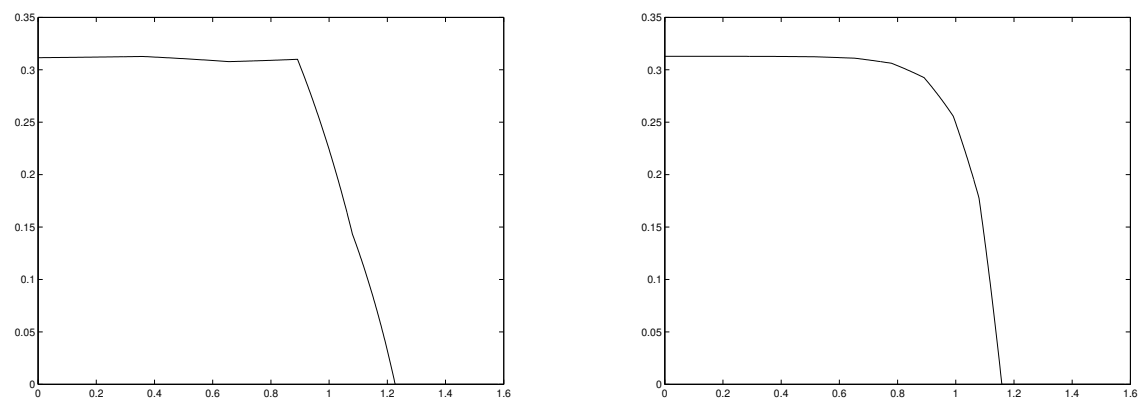

Figure 5: Non-uniform grid $n=16$ and $n=1024$

\section{References}

[1] N. Arada, E. Casas, and F. Tröltzsch. Error estimates for a semilinear elliptic optimal control problem. Computional Optimization and Approximation, 23:201-229, 2002.

[2] E. Casas and F. Tröltzsch. Error estimates for linear-quadratic elliptic control problems. In V. Barbu et al, editor, Analysis and Optimization of Differential Systems, pages 89-100, Boston, 2003. Kluwer Academic Publishers.

[3] R. Falk. Approximation of a class of optimal control problems with order of convergence estimates. J. Math. Anal. Appl., 44:28-47, 1973.

[4] T. Geveci. On the approximation of the solution of an optimal control problem governed by an elliptic equation. R.A.I.R.O. Analyse numeriqué, 13:313-328, 1979.

[5] W. Hager and G. Ianulescu. Dual approximation in optimal control. SIAM J. on Control and Optimization, 22:423-466, 1984.

[6] M. Heinkenschloss and F. Tröltzsch. Analysis of the Lagrange-SQP-Newton method for the control of a phase field equation. Control and Cybernetics, 28:178-211, 1999.

[7] M. Hinze. A generalized discretization concept for optimal control problems with control constraints. Technical Report MATH-NM-02-2003, TU Dresden, April 2003.

[8] C.T. Kelley and E. Sachs. Approximate quasi-Newton methods. Mathematical Programming, 48:41-70, 1990.

[9] K. Kunisch and A. Rösch. Primal-dual active set strategy for a general class of constrained optimal control problems. SIAM Journal Optimization, to appear. 
[10] K. Kunisch and E. Sachs. Reduced SQP-methods for parameter identification problems. SIAM Journal Numerical Analysis, 29:1793-1820, 1992.

[11] J.L. Lions and E. Magenes. Problèmes aux limites non homogènes et applications, volume 1-3. Dunod, Paris, 1968.

[12] K. Malanowski. Convergence of approximations vs. regularity of solutions for convex, control-constrained optimal control problems. Appl.Math.Opt., 8:6995, 1981.

[13] A. Rösch. Error estimates for linear-quadratic control problems with control constraints. Optimization Methods and Software, submitted.

[14] F. Tröltzsch. An SQP method for the optimal control of a nonlinear heat equation. Control and Cybernetics, 23(1/2):267-288, 1994. 